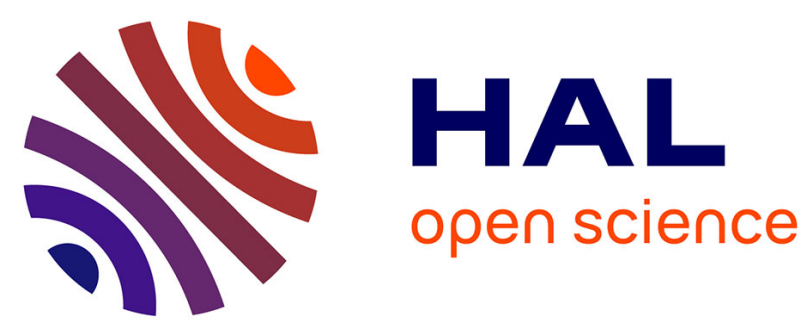

\title{
Bayesian update of the parameters of probability distributions for risk assessment in a two-level hybrid probabilistic-possibilistic uncertainty framework \\ Nicola Pedroni, Enrico Zio, Alberto Pasanisi, Mathieu Couplet
}

\section{- To cite this version:}

Nicola Pedroni, Enrico Zio, Alberto Pasanisi, Mathieu Couplet. Bayesian update of the parameters of probability distributions for risk assessment in a two-level hybrid probabilistic-possibilistic uncertainty framework. ESREL 2013, Sep 2013, Amsterdam, Netherlands. pp.1-8. hal-00839962

\section{HAL Id: hal-00839962}

https://hal-centralesupelec.archives-ouvertes.fr/hal-00839962

Submitted on 1 Jul 2013

HAL is a multi-disciplinary open access archive for the deposit and dissemination of scientific research documents, whether they are published or not. The documents may come from teaching and research institutions in France or abroad, or from public or private research centers.
L'archive ouverte pluridisciplinaire HAL, est destinée au dépôt et à la diffusion de documents scientifiques de niveau recherche, publiés ou non, émanant des établissements d'enseignement et de recherche français ou étrangers, des laboratoires publics ou privés. 


\title{
Bayesian update of the parameters of probability distributions for risk assessment in a two-level hybrid probabilistic-possibilistic uncertainty framework
}

\author{
N. Pedroni, E. Zio \\ Ecole Centrale Paris, Chatenay-Malabry, France \& Supelec, Gif-Sur-Yvette, France
}

A. Pasanisi, M. Couplet

Electricitè de France, Chatou, France

\begin{abstract}
Risk analysis models describing aleatory (i.e., random) events contain parameters (e.g., probabilities, failure rates, ...) that are epistemically uncertain, i.e., known with poor precision. Whereas probability distributions are always used to describe aleatory uncertainty, alternative frameworks of representation may be considered for describing epistemic uncertainty, depending on the information and data available.

In this paper, we use possibility distributions to describe the epistemic uncertainty in the parameters of the (aleatory) probability distributions.

We address the issue of updating, in a Bayesian framework, the possibilistic representation of the epistemically-uncertain parameters of (aleatory) probability distributions as new information (e.g., data) becomes available. A purely possibilistic counterpart of the classical, well-grounded probabilistic Bayes theorem is adopted.

The feasibility of the method is shown on a literature case study involving the risk-based design of a flood protection dike.
\end{abstract}

\section{INTRODUCTION}

We consider a framework of uncertainty representation with two hierarchical levels (Limbourg \& de Rocquigny 2010), in which risk analysis models of aleatory (i.e., random) events (e.g., failures) contain parameters (e.g., probabilities, failure rates, ...) that are epistemically-uncertain, i.e., known with poor precision due to lack of knowledge and information. Traditionally, both types of uncertainty are represented by probability distributions (USNRC 2009) and Bayes' rule is useful for updating the (probabilistic) epistemic uncertainty representation as new information (e.g., data) becomes available (Kelly \& Smith 2011).

However, in some situations, insufficient knowledge, information and data impairs a probabilistic representation of epistemic uncertainty. A number of alternative representation frameworks have been proposed for such cases, e.g., e.g., fuzzy set theory, evidence theory, possibility theory and interval analysis (Aven \& Zio 2011).

In this paper, we adopt possibility distributions to describe epistemic uncertainty (Baudrit \& Dubois 2006, Baudrit et al. 2008) and address the issue of updating, in a Bayesian framework, the possibilistic representation of the epistemically-uncertain parameters of (aleatory) probability distributions. We take an approach of literature based on a purely possibilistic counterpart of the classical, well- grounded probabilistic Bayes' theorem: it requires the construction of a possibilistic likelihood function which is used to revise the prior possibility distributions of the uncertain parameters (determined on the basis of a priori subjective knowledge and/or data) (Lapointe \& Bobee 2000). To the best of the authors' knowledge, this is the first time that the above mentioned technique is applied to risk assessment problems where hybrid uncertainty is separated into two hierarchical levels. To keep the analysis simple and retain a clear view of each step, the investigations are carried out with respect to a simple literature case study involving the risk-based design of a flood protection dike (Limbourg \& de Rocquigny 2010).

Other methods have been proposed in the literature to revise, in a Bayesian framework, nonprobabilistic representations of epistemic uncertainty. In (Stein et al. 2013) a modification of the Bayes' theorem is presented to account for the presence of fuzzy data and fuzzy prior PDFs. Finally, in (Smets 1993) a Generalized Bayes Theorem (GBT) is proposed within the framework of evidence theory: this approach is applied by (Le-Duy et al. 2011) to update the estimates of the failure rates of mechanical components in the context of nuclear Probabilistic Risk Assessment (PRA).

The remainder of the paper is organized as follows. In Section 2, the representation of aleatory (probabilistic) and epistemic (possibilistic) uncer- 
tainties in a "two-level" framework is provided; in Section 3, the method employed in this paper for the Bayesian update of the possibilistic parameters of aleatory probability distributions is described in details; in Section 4, the case study concerning the risk-based design of a flood protection dike is presented; in Section 5, the method of Section 3 is applied to the case study of Section 4; finally, some conclusions are drawn in the last Section 6.

\section{REPRESENTATION OF ALEATORY AND EPISTEMIC UNCERTAINTIES IN A TWO- LEVEL FRAMEWORK}

In all generality, we consider an uncertain variable $Y$, whose uncertainty is described by the Probability Distribution Function (PDF) $p^{Y}(y \mid \boldsymbol{\theta})$, where $\boldsymbol{\theta}=\left\{\theta_{1}, \theta_{2}, \ldots, \theta_{m}, \ldots, \theta_{P}\right\}$ is the vector of the corresponding internal parameters. In a two-level framework, the parameters $\boldsymbol{\theta}$ are themselves affected by epistemic uncertainty (Limbourg \& de Rocquigny 2010). In the present work, we describe these uncertainties by the (generally joint) possibility distribution $\pi^{\theta}(\boldsymbol{\theta})$ (Baudrit et al. 2008).

For clarification by way of example, we may consider the generic uncertain variable $Y$ described by a Gumbel PDF, i.e., $Y \sim p^{Y}(y \mid \boldsymbol{\theta})=\operatorname{Gum}(\boldsymbol{\theta})=$ $\operatorname{Gum}\left(\theta_{1}, \theta_{2}\right)=\operatorname{Gum}(\gamma, \delta)=p^{Y}(y \mid \gamma, \delta)$. Parameter $\delta=\theta_{2}$ is a fixed point-wise value $\left(\delta=\theta_{2}=100\right)$, whereas parameter $\gamma=\theta_{1}$ is epistemically-uncertain. By hypothesis, the only information available on $\gamma=\theta_{1}$ is that it is defined on interval $\left[a_{\gamma}, b_{\gamma}\right]=[900$, 1300] and its most likely value is $c_{\gamma}=1100$. This limited state of knowledge about $\gamma=\theta_{1}$ can be described by a triangular possibility distribution $\pi^{\gamma}(\gamma)$ with core $c_{\gamma}=1100$ and support $\left[a_{\gamma}, b_{\gamma}\right]=[900$, 1300] (Figure 1, top) (Baudrit \& Dubois 2006).

Given the possibility distribution of $\gamma=\theta_{1}$, we can define its $\alpha$-cut sets $A_{\alpha}^{\gamma}=\left\{\gamma: \pi^{\gamma}(\gamma) \geq \alpha\right\}$, with $0 \leq \alpha \leq 1$. For example, $A_{0.5}^{\gamma}=[1000,1200]$ is the set of $\gamma$ values for which the possibility function is greater than or equal to 0.5 (dashed segment in Figure 1, top). Notice that the $\alpha$-cut set $A_{\alpha}^{\gamma}$ of parameter $\gamma$ can be interpreted also as the $(1-\alpha) \cdot 100 \%$ Confidence Interval (CI) for $\gamma$, i.e., the interval such that $P\left[\gamma \in A_{\alpha}^{\gamma}\right] \geq 1-\alpha$. For example, $A_{0}^{\gamma}=[900$, $1300]$ is the $(1-0) \cdot 100 \%=100 \%$ CI for $\gamma$, i.e., the interval that contains the "true" value of $\gamma$ with certainty (solid segment in Figure 1, top); $A_{0.5}^{\gamma}=[1000$, 1200] $\left(\subset A_{0}^{\gamma}\right)$ is the $(1-0.5) \cdot 100 \%=50 \% \mathrm{CI}$ (dashed segment in Figure 1, top) and so on. In this view, the possibility distribution $\pi^{\gamma}(\gamma)$ can be interpreted as a set of nested CIs for parameter $\gamma$ (Baudrit \& Dubois 2006).

For each possibility (resp., confidence) level $\alpha$ (resp., $1-\alpha$ ) in $[0,1]$, a bundle of Cumulative Distribution Functions (CDFs) for $Y$, namely $\left\{F^{Y}(y \mid \gamma, \delta)\right\}_{\alpha}$, can be generated by letting the epistemically-uncertain parameter $\gamma$ range within the corresponding $\alpha$-cut set $A_{\alpha}^{\gamma}$, i.e., $\left\{F^{Y}(y \mid \gamma, \delta)\right\}_{\alpha}=$ $\left\{F^{Y}(y \mid \gamma, \delta): \gamma \in A_{\alpha}^{\gamma}, \sigma=100\right\}$. This family of CDFs (of level $\alpha$ ) is bounded above and below by the upper and lower CDFs, $\bar{F}_{\alpha}^{Y}(y)$ and $\underline{F}_{\alpha}^{Y}(y)$, defined as $\bar{F}_{\alpha}^{Y}(y)=\sup _{\gamma \in A_{\alpha}^{\gamma}}\left\{F^{Y}(y \mid \gamma, \delta=100)\right\}$ and $\underline{F}_{\alpha}^{Y}(y)=$ $\inf _{\gamma \in A_{\alpha}^{\gamma}}\left\{F^{Y}(y \mid \gamma, \delta=100)\right\}$, respectively. Since $\pi^{\gamma}(\gamma)$ can be interpreted as a set of nested CIs for parameter $\gamma$ (see above), it can be argued that the $\alpha$-cuts of $\pi^{\gamma}(\gamma)$ induce also a set of nested pairs of CDFs $\left\{\left(\underline{F}_{\alpha}^{Y}(y), \bar{F}_{\alpha}^{Y}(y)\right): 0 \leq \alpha \leq 1\right\}$ which bound the "true" CDF $F^{Y}(y)$ of $Y$ with confidence larger than or equal to $(1 \quad-\quad-\alpha)$, i.e., $P\left[\underline{F}_{\alpha}^{Y}(y) \leq F^{Y}(y) \leq \bar{F}_{\alpha}^{Y}(y)\right] \geq 1-\alpha$, with $0 \leq \alpha \leq 1$ (Baudrit et al. 2008). In passing, notice that the upper and lower CDFs (of level $\alpha$ ), $\bar{F}_{\alpha}^{Y}(y)$ and $\underline{F}_{\alpha}^{Y}(y)$, can be referred to as the plausibility and belief functions (of level $\alpha$ ) of the set $Z=(-\infty, y]$, i.e., $\bar{F}_{\alpha}^{Y}(y)=P l_{\alpha}^{Y}(Z)$ and $\underline{F}_{\alpha}^{Y}(y)=\operatorname{Bel}_{\alpha}^{Y}(Z)$, respectively. For illustration purposes, Figure 1, bottom, shows the bounding upper and lower CDFs of $Y, P l_{\alpha}^{Y}(Z)$ and $\operatorname{Bel}_{\alpha}^{Y}(Z)$, built in correspondence of the $\alpha$-cuts of level $\alpha=0$ (solid lines), 0.5 (dashed lines) and 1 (dot-dashed line) of the possibility distribution $\pi^{\gamma}(\gamma)$ of parameter $\gamma$ (Figure 1, top).

Finally, the set of nested pairs of CDFs $\left\{\left(\operatorname{Bel}_{\alpha}^{Y}(Z), P l_{\alpha}^{Y}(Z)\right): 0 \leq \alpha \leq 1\right\}, Z=(-\infty, y]$, can be synthesized into a single pair of plausibility and belief functions as $P l^{Y}(Z)=\int_{0}^{1} P l_{\alpha}^{Y}(Z) d \alpha \quad$ and $\operatorname{Bel}^{Y}(Z)=\int_{0}^{1} \operatorname{Bel}_{\alpha}^{Y}(Z) d \alpha$, respectively (dotted lines in Figure 1, bottom). The plausibility and belief functions $\mathrm{Pl}^{Y}(Z)$ and $\mathrm{Bel}^{Y}(Z), Z=(-\infty, y]$, are shown to represent the "best bounds" for the "true" CDF 
$F^{Y}(y)$ of the uncertain variable $Y$ (Baudrit et al. 2008).

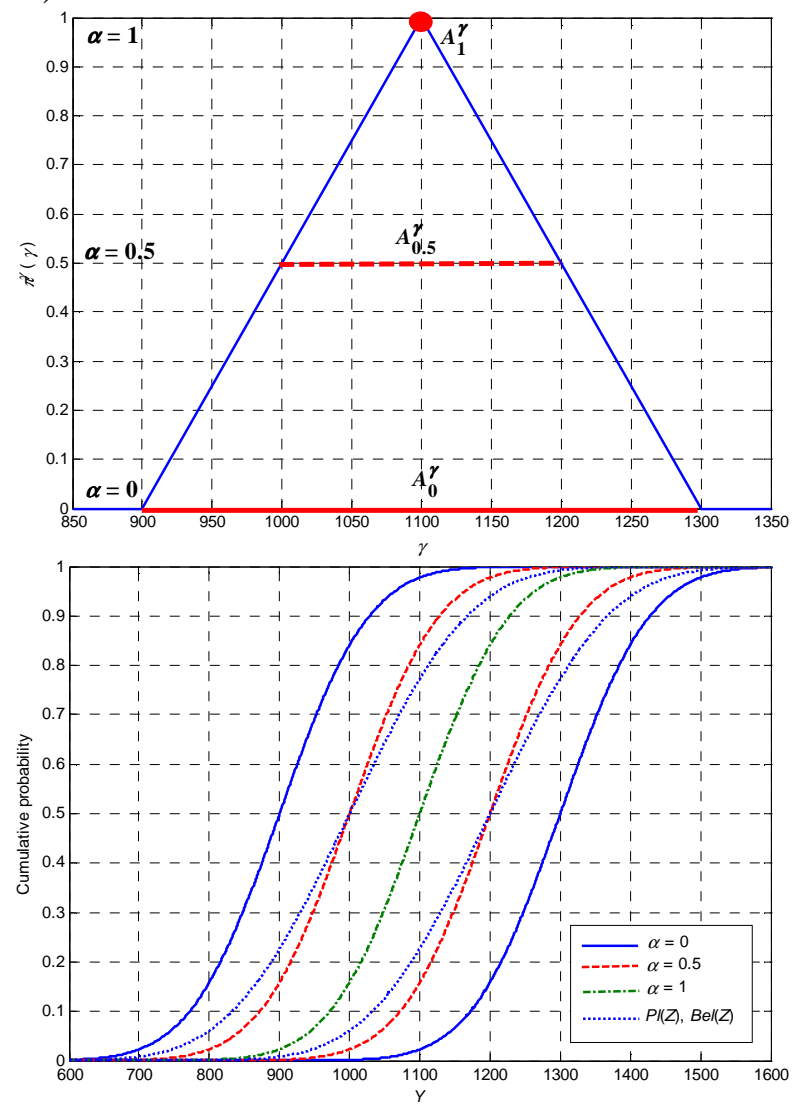

Figure 1. Top: triangular possibility distribution $\pi^{\gamma}(\gamma)$ of the uncertain parameter $\gamma$ of the Gumbel PDF of $Y \sim$ $p^{Y}(y \mid \gamma, \delta=100)$; in evidence the $\alpha$-cuts of level $\alpha=0$ (solid segment), 0.5 (dashed segment) and 1 (dot). Bottom: bounding upper and lower CDFs of $Y, P l_{\alpha}^{Y}(Z)$ and $\operatorname{Bel}_{\alpha}^{Y}(Z), Z=(-\infty, y]$, built in correspondence of the $\alpha$-cuts of level $\alpha=0$ (solid lines), 0.5 (dashed lines) and 1 (dot-dashed line) of $\pi^{\gamma}(\gamma)$; the plausibility and belief functions $\operatorname{Pl}^{Y}(Z)$ and $\operatorname{Bel}^{Y}(Z), Z=(-\infty, y]$, are also shown (dotted lines)

\section{BAYESIAN UPDATE OF THE POSSIBILISTIC PARAMETERS OF ALEATORY \\ PROBABILITY DISTRIBUTIONS}

Let $\pi^{\theta}(\boldsymbol{\theta})$ be the (joint) prior possibility distribution for the parameters $\boldsymbol{\theta}=\left[\theta_{1}, \theta_{2}, \ldots, \theta_{m}, \ldots, \theta_{P}\right]$ of the PDF $p^{Y}(y \mid \boldsymbol{\theta})$ of variable $Y$ (built on the basis of a priori subjective engineering knowledge and/or data). For example, in the risk assessment context of this paper $Y$ may represent the yearly maximal water flow of a river described by a Gumbel distribution: thus, $Y \sim p^{Y}(y \mid \boldsymbol{\theta})=\operatorname{Gum}(\boldsymbol{\theta})=\operatorname{Gum}\left(\theta_{1}, \theta_{2}\right)=$ $\operatorname{Gum}(\gamma, \delta)=p^{Y}(y \mid \gamma, \delta)$ and $\pi^{\theta}(\boldsymbol{\theta})=\pi^{\gamma, \delta}(\gamma, \delta)$. Moreover, let $\boldsymbol{y}=\left[y_{1}, y_{2}, \ldots, y_{k}, \ldots, y_{D}\right]$ be a vector of $D$ observed pieces of data representing the new information/evidence available for the analysis: referring to the example above, $\boldsymbol{y}$ may represent a vector of $D$ values collected over a long period time (e.g., many years) of the yearly maximal water flow of the river under analysis. The objective of the Bayesian analysis is to update the a priori representation $\pi^{\theta}(\boldsymbol{\theta})=\pi^{\gamma, \delta}(\gamma, \boldsymbol{\delta})$ of $\boldsymbol{\theta}=[\gamma, \delta]$ on the basis of the new evidence acquired, i.e., to calculate the posterior possibility distribution $\pi^{\theta}(\boldsymbol{\theta} \mid \boldsymbol{y}) \quad$ (i.e., $\left.\pi^{\gamma, \delta}(\gamma, \delta \mid \boldsymbol{y})\right)$ of $\boldsymbol{\theta}$ after $\boldsymbol{y}$ is obtained.

The method considered in this paper is based on a purely possibilistic counterpart of the classical, probabilistic Bayes' theorem (Lapointe \& Bobée 2000):

$$
\pi^{\theta}(\boldsymbol{\theta} \mid \boldsymbol{y})=\frac{\pi_{L}^{\theta}(\boldsymbol{\theta} \mid \boldsymbol{y}) \cdot \pi^{\theta}(\boldsymbol{\theta})}{\sup _{\boldsymbol{\theta}}\left\{\pi_{L}^{\theta}(\boldsymbol{\theta} \mid \boldsymbol{y}) \cdot \pi^{\theta}(\boldsymbol{\theta})\right\}},
$$

where $\pi_{L}^{\theta}(\boldsymbol{\theta} \mid \boldsymbol{y})$ is the possibilistic likelihood of the parameter vector $\boldsymbol{\theta}$ given the newly observed data $\boldsymbol{y}$, and quantities $\pi^{\theta}(\boldsymbol{\theta} \mid \boldsymbol{y})$ and $\pi^{\theta}(\boldsymbol{\theta})$ are defined above. Notice that $\sup _{\boldsymbol{\theta}}\left\{\pi_{L}^{\theta}(\boldsymbol{\theta} \mid \boldsymbol{y}) \cdot \pi^{\theta}(\boldsymbol{\theta})\right\}$ is a normalization factor such that $\sup _{\boldsymbol{\theta}}\left\{\pi^{\theta}(\boldsymbol{\theta} \mid \boldsymbol{y})\right\}=1$, as required by possibility theory (Baudrit \& Dubois 2006).

It is worth mentioning that forms of the possibilistic Bayes' theorem alternative to (1) can be constructed as a result of other definitions of the operation of 'conditioning' with possibility distributions: the reader is referred to (Lapointe \& Bobée 2000) for technical details. In this paper, expression (1) has been chosen because "it satisfies desirable properties of the revision process and lead to continuous posterior distributions" (Lapointe \& Bobée 2000).

The possibilistic likelihood $\pi_{L}^{\theta}(\boldsymbol{\theta} \mid \boldsymbol{y})$ is here obtained by transforming the classical probabilistic likelihood function $L^{\theta}(\boldsymbol{\theta} \mid \boldsymbol{y})$ through normalization, i.e., $\pi_{L}^{\theta}(\boldsymbol{\theta} \mid \boldsymbol{y})=L^{\theta}(\boldsymbol{\theta} \mid \boldsymbol{y}) / \sup _{\boldsymbol{\theta}}\left\{L^{\theta}(\boldsymbol{\theta} \mid \boldsymbol{y})\right\}$. This choice has been made for the following main reasons: (i) the transformation is simple and can be straightforwardly applied to any distribution; (ii) the resulting possibilistic likelihood is very closely related to the classical, purely probabilistic one (which is theoretically well-grounded) by means of the simple and direct operation of normalization that preserves the "original structure" of the experimental evidence; (iii) it can be easily verified that the resulting possibilistic likelihood keeps the sequential nature of the updating procedure typical of the standard Bayes' theorem. On the other hand, it has to be also admitted that the resulting possibility distributions 
do not in general adhere to the probability-possibility consistency principle (Baudrit \& Dubois 2006).

It is worth noting that other techniques of transformation of probability density functions into possibility distributions exist, but the corresponding details are not given here for brevity sake: the interested reader is referred to (Flage et al. 2013) for some proposed techniques, e.g., the principle of maximum specificity and the principle of minimal commitment. Also, it has to be noticed that other techniques are available to construct possibility distributions (and, thus, possibilistic likelihood functions) directly from rough experimental data (i.e., without resorting to probability-possibility transformations): see, e.g., (Serrurier \& Prade 2011).

It is worth noting that the application of the approach always produces a joint $P$-dimensional posterior possibility distribution $\pi^{\theta}(\boldsymbol{\theta} \mid \boldsymbol{y})$ (whatever the state of dependence between the priors), characterized by $P$-dimensional $\alpha$-cuts $A_{\alpha}^{\theta \mid y}$, with $0<\alpha<1$ : as a consequence, there is an interactive dependence between the values that parameters $\left\{\theta_{m}: m=1,2, \ldots\right.$, $P$ \} can take when ranging within a given $\alpha$-cut $A_{\alpha}^{\theta \mid y}$. From $\pi^{\theta}(\boldsymbol{\theta} \mid \boldsymbol{y})$ it is straightforward to obtain the marginal posterior possibility distribution $\pi^{\theta_{m}}\left(\theta_{m} \mid \boldsymbol{y}\right)$ for each parameter $\theta_{m}$ as $\pi^{\theta_{m}}\left(\theta_{m} \mid \boldsymbol{y}\right)=$ $\max _{\theta_{j} \in \Re, j \neq m}\left\{\pi^{\theta}(\boldsymbol{\theta} \mid \boldsymbol{y})\right\}, \quad \forall \theta_{m} \in \mathfrak{R}, \quad m=1,2, \ldots, P:$ $\pi^{\theta_{m}}\left(\theta_{m} \mid \boldsymbol{y}\right)$ is the projection of $\pi^{\boldsymbol{\theta}}(\boldsymbol{\theta} \mid \boldsymbol{y})$ onto the $m$ th axis. The (one-dimensional) $\alpha$-cut $A_{\alpha}^{\theta_{m} \mid y}=$ $\left[\underline{\theta}_{m, \alpha}\left|y, \bar{\theta}_{m, \alpha}\right| y\right]$ of the marginal possibility distribution $\pi^{\theta_{m}}\left(\theta_{m} \mid \boldsymbol{y}\right)$ is then related to the $(P-$ dimensional) $\alpha$-cut $A_{\alpha}^{\theta \mid y}$ of the joint possibility distribution $\pi^{\theta}(\boldsymbol{\theta} \mid \boldsymbol{y})$ by the following straightforward relation, i.e., $A_{\alpha}^{\theta_{m} \mid y}=\left[\underline{\theta}_{m, \alpha}\left|y, \bar{\theta}_{m, \alpha}\right| y\right]=$ $\left[\min _{\boldsymbol{\theta} \in A_{\alpha}^{\theta \mid y}}\left\{\theta_{m}\right\}, \max _{\boldsymbol{\theta} \in A_{\alpha}^{\mid l y}}\left\{\theta_{m}\right\}\right]$. In this view, notice that the use of the $P$-dimensional $\alpha$-cut $A_{\alpha, \text { Cart }}^{\theta \mid y}$ constructed by the Cartesian product of the (one-dimensional) $\alpha$ cuts $A_{\alpha}^{\theta_{m} \mid y}$ of the marginal distributions, $m=1,2, \ldots$, $P$ (i.e., $A_{\alpha, \text { Cart }}^{\theta \mid y}=A_{\alpha}^{\theta_{1} \mid y} \times A_{\alpha}^{\theta_{2} \mid y} \times \ldots \times A_{\alpha}^{\theta_{m} \mid y} \times \ldots \times$ $A_{\alpha}^{\theta_{p} \mid y}$ ) would (incorrectly) imply independence between the posterior estimates of the parameters $\left\{\theta_{m}\right.$ : $m=1,2, \ldots, P\}$; however, since $A_{\alpha, \text { Cart }}^{\theta \mid y}$ completely contains $A_{\alpha}^{\theta \mid y}$ (i.e., by definition $A_{\alpha}^{\theta \mid y} \subset A_{\alpha, \text { Cart }}^{\theta \mid y}$ ), then conservatism would be still guaranteed (Stein et al. 2013).

\section{CASE STUDY: FLOOD PROTECTION RISK- BASED DESIGN}

The maximal water level of the river (i.e., the output variable of the model, $Z_{c}$ ) is given as a function of several (and some uncertain) parameters (i.e., the inputs to the model) (Limbourg \& de Rocquigny 2010):

$$
Z_{c}=f\left(Q, Z_{m}, Z_{v}, K_{s}, B, L\right)=f\left(Y_{1}, Y_{2}, Y_{3}, Y_{4}, Y_{5}, Y_{6}\right)
$$

where: $Y_{1}=Q$ is the yearly maximal water discharge $\left[\mathrm{m}^{3} / \mathrm{s}\right] ; Y_{2}=Z_{m}$ and $Y_{3}=Z_{v}$ are the riverbed levels [m asl] at the upstream and downstream parts of the river under investigation, respectively; $Y_{4}=$ $K_{s}$ is the Strickler friction coefficient; $Y_{5}=B$ and $Y_{6}=L$ are the width and length of the river part $[\mathrm{m}]$, respectively. Quantities $Y_{5}=B(=300 \mathrm{~m})$ and $Y_{6}=L$ $(=5000 \mathrm{~m})$ are constant parameters, whereas quantities $Y_{1}=Q, Y_{2}=Z_{m}, Y_{3}=Z_{v}, Y_{4}=K_{s}$ are uncertain variables.

The $n=4$ input variables $Y_{i}, i=1,2,3,4$, are affected by aleatory and epistemic uncertainties. The aleatory part of the uncertainty is described by probability distributions of defined shape. The parameters of the probability distributions describing the aleatory uncertainty are themselves affected by epistemic uncertainty and represented in terms of possibility distributions.

The aleatory uncertainty in the yearly maximal water flow $Y_{1}=Q$ is well described by a Gumbel probability distribution $p^{Q}(q \mid \gamma, \delta)=\operatorname{Gum}(\gamma, \delta)=$ $\frac{1}{\delta} \exp \left[-\exp \left(\frac{\gamma-q}{\delta}\right)\right] \exp \left[\frac{\gamma-q}{\delta}\right]$ (Limbourg \& de Rocquigny 2010). The extreme physical bounds on variable $Q$ are $Q_{\min }=10 \mathrm{~m}^{3} / \mathrm{s}$ (which is a typical drought flow level) and $Q_{\max }=10000 \mathrm{~m}^{3} / \mathrm{s}$ (which is three times larger than the maximal flood ever occurred). The prior possibility distributions $\pi^{\gamma}(\gamma)$ and $\pi^{\delta}(\delta)$ for the epistemically-uncertain parameters $\gamma$ and $\delta$ are subjectively chosen as triangular functions $\operatorname{TR}\left(a_{\gamma}, c_{\gamma}, b_{\gamma}\right)$ and $\operatorname{TR}\left(a_{\delta}, c_{\delta}, b_{\delta}\right)$, respectively, with cores (i.e., preferred or most likely values) $c_{\gamma}$ $=955 \mathrm{~m}^{3} / \mathrm{s}$ and $c_{\delta}=600 \mathrm{~m}^{3} / \mathrm{s}$, and supports $\left[a_{\gamma}, b_{\gamma}\right]=$ $[869,1157] \mathrm{m}^{3} / \mathrm{s}$ and $\left[a_{\delta}, b_{\delta}\right]=[455,660] \mathrm{m}^{3} / \mathrm{s}$, respectively. The Bayesian update of these uncertainty representations (based on prior subjective knowledge) is realized with the aid of a vector $\boldsymbol{y}_{1}=\boldsymbol{q}$ $=\left[q_{1}, q_{2}, \ldots, q_{k}, \ldots, q_{149}\right]$ of $D_{1}=149$ (independent and identically distributed - iid) values of the annual maximal flow of the river. The point estimates $\hat{\gamma}^{M L E}$ and $\hat{\delta}^{M L E}$ for $\gamma$ and $\delta$ obtained by the classical, purely probabilistic Maximum Likelihood Estimation (MLE) method are $1013.21 \mathrm{~m}^{3} / \mathrm{s}$ and 558.21 $\mathrm{m}^{3} / \mathrm{s}$, respectively. 
The aleatory part of the uncertainty in the upstream riverbed level $Y_{2}=Z_{m}$ is represented by a normal distribution, i.e., $Z_{m} \sim p^{Z_{m}}\left(z_{m} \mid \mu_{Z m}, \sigma_{Z m}\right)=$ $N\left(\mu_{Z m}, \sigma_{Z m}\right)$ (Limbourg \& de Rocquigny 2010). This distribution is truncated at the minimum and maximum physical bounds on $Z_{m}$, i.e., $Z_{m, \text { min }}=53.5 \mathrm{~m}$ (given by plausible lower geomorphologic limits to erosion) and $Z_{m, \max }=57 \mathrm{~m}$ (given by plausible upper geomorphologic limits to sedimentation), respectively. The prior possibility distributions for $\mu_{Z m}$ and $\sigma_{Z m}$ are the triangular functions $\pi^{\mu_{Z n}}\left(\mu_{Z m}\right)=\operatorname{TR}\left(a_{\mu_{Z m}}, \quad c_{\mu_{Z m}}, \quad b_{\mu_{Z m}}\right)=\operatorname{TR}(54.78$, $54.93,55.28)$ and $\pi^{\sigma_{Z n}}\left(\sigma_{Z m}\right)=\operatorname{TR}\left(a_{\sigma_{Z m}}, c_{\sigma_{Z m}}, b_{\sigma_{Z m}}\right)$ $=T R(0.33,0.51,0.58)$, respectively. The Bayesian update of these uncertainty representations is carried out using a vector $\boldsymbol{y}_{2}=z_{\boldsymbol{m}}=\left[z_{m, 1}, z_{m, 2}, \ldots, z_{m, k}, \ldots\right.$, $\left.z_{m, 29}\right]$ of $D_{2}=29$ (iid) values of the upstream riverbed level. The MLE estimates of the parameters are $\hat{\mu}_{Z m}^{M L E}=50.19 \mathrm{~m}$ and $\hat{\sigma}_{Z m}^{M L E}=0.38 \mathrm{~m}$, respectively.

As for $Y_{2}=Z_{m}$, the aleatory part of the uncertainty in the downstream riverbed level $Y_{3}=Z_{v}$ is represented by a normal distribution, i.e., $Z_{v} \sim$ $p^{Z_{v}}\left(z_{v} \mid \mu_{Z v}, \sigma_{Z v}\right)=N\left(\mu_{Z v}, \sigma_{Z v}\right)$, truncated at $Z_{v \text {,min }}=48 \mathrm{~m}$ and $Z_{v, \text { max }}=51 \mathrm{~m}$. As before, the prior possibility distributions are triangular functions, i.e., $\pi^{\mu_{Z v}}\left(\mu_{Z v}\right)=T R\left(a_{\mu_{z v}}, c_{\mu_{z v}}, b_{\mu_{Z v}}\right)=T R(49.98,50.11$, $50.40)$ and $\pi^{\sigma_{Z v}}\left(\sigma_{Z v}\right)=\operatorname{TR}\left(a_{\sigma_{Z v}}, c_{\sigma_{Z v}}, b_{\sigma_{Z v}}\right)=$ $\operatorname{TR}(0.23,0.45,0.54)$. These representations are updated by means of a vector $\boldsymbol{y}_{3}=\boldsymbol{z}_{v}=\left[z_{v, 1}, z_{v, 2}, \ldots, z_{v, k}\right.$, $\left.\ldots, z_{v, 29}\right]$ of $D_{3}=29$ (iid) values of the downstream riverbed level. The MLE estimates of the parameters are $\hat{\mu}_{Z v}^{M L E}=55.03 \mathrm{~m}$ and $\hat{\sigma}_{Z v}^{M L E}=0.45 \mathrm{~m}$, respectively.

The Strickler friction coefficient $Y_{4}=K_{s}$ is the most critical source of uncertainty because it is usually a simplification of a complex hydraulic model. The absolute physical limits of $K_{s}$ are 5 and 60, respectively (Limbourg \& de Rocquigny 2010). The friction coefficient $K_{s}$ is affected by random events modifying the river status (e.g., erosion, sedimentation, ...): the corresponding variability is typically described by a normal distribution, i.e., $K_{s} \sim$ $p^{K_{s}}\left(k_{s} \mid \mu_{K s}, \sigma_{K s}\right)=N\left(\mu_{K s}, \sigma_{K s}\right)$ (Limbourg \& de Rocquigny 2010). However, the parameters of this normal distribution are difficult to estimate because data can only be obtained through "indirect calibration characterized by significant uncertainty" (Limbourg \& de Rocquigny 2010): the uncertainty in these parameters is described by triangular possibility distributions. The possibilistic functions $\pi^{\mu_{K_{s}}}\left(\mu_{K_{s}}\right)$ and $\pi^{\sigma_{K s}}\left(\sigma_{K s}\right)$ used to represent the a priori knowledge on $\mu_{K s}$ and $\sigma_{K s}$ are $\operatorname{TR}\left(a_{\mu_{K s}}, c_{\mu_{K s}}\right.$, $\left.b_{\mu_{K s}}\right)=\operatorname{TR}(21.37,25.23,34.23)$ and $\operatorname{TR}\left(a_{\sigma_{K s}}, c_{\sigma_{K s}}\right.$, $\left.b_{\sigma_{K s}}\right)=T R(1.16,6.91,9.37)$, respectively. The Bayesian revision of these a priori representations is performed by means of a vector $\boldsymbol{y}_{4}=\boldsymbol{k}_{\boldsymbol{s}}=\left[k_{s, 1}, k_{s, 2}\right.$, $\left.\ldots, k_{s, k}, \ldots, k_{s, 5}\right]$ of $D_{4}=5$ (iid) values of the Strickler friction coefficient. The MLE estimates of the parameters are $\hat{\mu}_{K s}^{M L E}=27.8$ and $\hat{\sigma}_{K s}^{M L E}=5.26$, respectively.

\section{RESULTS}

In order to simplify the notation, in what follows let $\theta$ be one of the uncertain parameters of the PDFs of $Y_{1}=Q, Y_{2}=Z_{m}, Y_{3}=Z_{v}$ and $Y_{4}=K_{s}$, i.e., $\theta=\gamma, \delta$, $\mu_{Z m}, \sigma_{Z m}, \mu_{Z v}, \sigma_{Z v}, \mu_{K_{s}}$ or $\sigma_{K s}$. Figure 2 illustrates the possibility distributions of the epistemicallyuncertain parameters of the aleatory PDFs $p^{Q}(q \mid \gamma, \delta)$ (top) and $p^{K_{s}}\left(k_{s} \mid \mu_{K s}, \sigma_{K s}\right)$ (bottom) of the uncertain input variables $Y_{1}=Q$ and $Y_{4}=K_{s}$, respectively; the parameters of the PDFs $p^{Z_{m}}\left(z_{m} \mid \mu_{Z m}, \sigma_{Z m}\right)$ and $p^{Z_{v}}\left(z_{v} \mid \mu_{Z v}, \sigma_{Z v}\right)$ of $Y_{2}=Z_{m}$ and $Y_{3}=Z_{v}$, respectively, are not shown due to space limitations. In particular, the prior possibility distributions $\pi^{\theta}(\theta) \quad\left(=\pi^{\gamma}(\gamma)\right.$, $\pi^{\delta}(\delta), \pi^{\mu_{K_{s}}}\left(\mu_{K_{s}}\right)$ and $\left.\pi^{\sigma_{K s}}\left(\sigma_{K s}\right)\right)$ are shown as solid lines, whereas the marginal posterior possibility distributions $\pi^{\theta}(\theta \mid \boldsymbol{y})\left(=\pi^{\gamma}(\gamma \mid \boldsymbol{q}), \pi^{\delta}(\delta \mid \boldsymbol{q})\right.$, $\pi^{\mu_{K_{s}}}\left(\mu_{K_{s}} \mid \boldsymbol{k}_{s}\right)$ and $\left.\pi^{\sigma_{K s}}\left(\sigma_{K s} \mid \boldsymbol{k}_{s}\right)\right)$ obtained using $D_{1}$ $=149$ and $D_{4}=5$ pieces of data are shown in dashed lines, respectively; the point estimates $\hat{\theta}^{M L E}$ (= $\hat{\gamma}^{M L E}, \hat{\delta}^{M L E}, \hat{\mu}_{K_{s}}^{M L E}$ and $\hat{\sigma}_{K s}^{M L E}$ ) produced by the classical MLE method are also shown for comparison (dots) (see Section 4). 

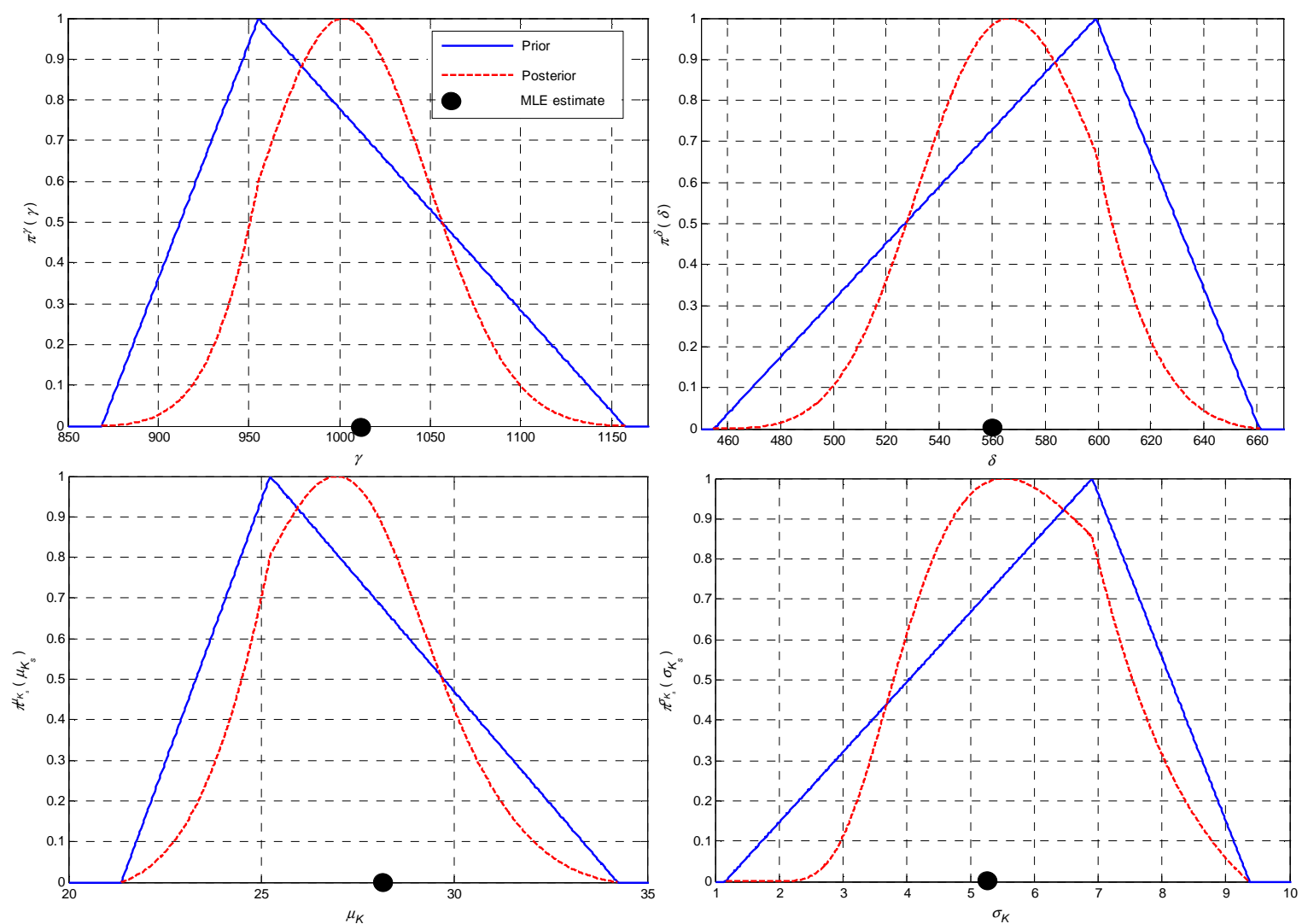

Figure 2. Possibility distributions of the epistemically-uncertain parameters of the aleatory $\operatorname{PDFs} p^{Q}(q \mid \gamma, \delta)$ (top) and $p^{K_{s}}\left(k_{s} \mid \mu_{K s}, \sigma_{K s}\right)$ (bottom) of the uncertain input variables $Y_{1}=Q$ and $Y_{4}=K_{s}$, respectively. Solid lines: priors; dashed lines: marginal posteriors. The point estimates of the parameters obtained by the classical MLE method are also shown for comparison (dots)

From a mere visual and qualitative inspection of Figure 2 it can be seen that the approach is suitable for revising the prior possibility distributions (based on a priori purely subjective knowledge) by means of empirical data. In particular, it is evident that: (i) the most likely (i.e., preferred) values $c_{\theta}$ of the epistemically-uncertain parameters (i.e., those values in correspondence of which the possibility function equals 1) are moved towards the MLE estimates $\hat{\theta}^{M L E}$ in all the cases considered; (ii) the area $S_{\theta}$ underlying the corresponding possibility distributions is significantly reduced: noting that this area is related to the imprecision in the knowledge of the possibilistic parameter (i.e., the larger the area, the higher the imprecision), it can be concluded that the approach succeeds in reducing the epistemic uncertainty. With respect to that, Table 1 reports the most likely values $c_{\theta}$ and the areas $S_{\theta}$ underlying the (marginal) possibility distributions of the uncertain parameter $\theta\left(=\gamma, \delta, \mu_{Z m}, \sigma_{Z m}, \mu_{Z v}, \sigma_{Z v}, \mu_{K_{s}}\right.$ and $\left.\sigma_{K s}\right)$ before and after the Bayesian update; the point estimates $\hat{\theta}^{M L E}$ obtained by the classical MLE method are also reported for completeness. In addition, in order to provide a more quantitative assessment of the "updating power" of the method, two indicators are defined: (i) the percentage relative difference $R_{\theta}^{\text {dist }}$ of the absolute distances, $d_{\theta}^{\text {Prior }}$ and $d_{\theta}^{\text {Posterior }}$, between the prior and posterior most likely values, $c_{\theta}^{\text {Prior }}$ and $c_{\theta}^{\text {Posterior }}$, respectively, of parameter $\theta$ and the corresponding MLE estimate $\hat{\theta}^{M L E}$, i.e.:

$R_{\theta}^{\text {dist }}=\left(d_{\theta}^{\text {Prior }}-d_{\theta}^{\text {Posterior }}\right) / d_{\theta}^{\text {Prior }} \cdot 100$,

where $d_{\theta}^{\text {Prior }}=\left|c_{\theta}^{\text {Prior }}-\hat{\theta}^{M L E}\right| / \hat{\theta}^{M L E}$ and $d_{\theta}^{\text {Posterior }}=$ $\left|c_{\theta}^{\text {Posterior }}-\hat{\theta}^{M L E}\right| / \hat{\theta}^{M L E}$. Obviously, the higher is $R_{\theta}^{\text {dist }}$ (i.e., the lower is $d_{\theta}^{\text {Posterior }}$ ) the closer is the most likely value $c_{\theta}^{\text {Posterior }}$ to the MLE estimate $\hat{\theta}^{M L E}$, i.e., the higher is the strength of the approach in updating the prior possibilistic distribution;

(ii) the percentage relative difference $R_{\theta}$ between the areas underlying the possibility distribution of parameter $\theta$ before and after the Bayesian update, namely $S_{\theta}^{\text {Prior }}$ and $S_{\theta}^{\text {Posterior }}$, respectively:

$R_{\theta}=\left(S_{\theta}^{\text {Prior }}-S_{\theta}^{\text {Posterior }}\right) / S_{\theta}^{\text {Prior }} \cdot 100$.

Again, the higher is $R_{\theta}$, the higher is the reduction in the area (i.e., in the epistemic uncertainty) and, thus, the higher is the "updating strength" of the approach. 
Table 1. Most likely values $c_{\theta}$ of the parameters $\theta=\gamma, \delta, \mu_{\mathrm{Zm}}, \sigma_{\mathrm{Zm}}, \mu_{\mathrm{Zv}}, \sigma_{\mathrm{Zv}}, \mu_{K_{s}}$ and $\sigma_{K s}$ of the aleatory PDFs of $Y_{1}=Q, Y_{2}=$ $Z_{m}, Y_{3}=Z_{v}$ and $Y_{4}=K_{s}$ and areas $S_{\theta}$ underlying the corresponding (marginal) possibility distributions before and after the Bayesian update. The point estimates $\hat{\theta}^{M L E}$ are also shown for comparison together with the values of $R_{\theta}^{\text {dist }}$ (3) and $R_{\theta}$ (4) (parentheses)

\begin{tabular}{|c|c|c|c|c|c|c|}
\hline \multirow[b]{3}{*}{$\boldsymbol{Y}_{j}$} & \multirow[b]{3}{*}{$\boldsymbol{\theta}$} & \multirow[b]{3}{*}{$\hat{\boldsymbol{\theta}}^{M L E}$} & \multicolumn{4}{|c|}{ Possibility distributions update } \\
\hline & & & \multicolumn{2}{|c|}{$c_{\theta}\left(\boldsymbol{R}_{\theta}^{\text {dist }}\right)$} & \multicolumn{2}{|c|}{$S_{\theta}\left(R_{\theta}\right)$} \\
\hline & & & Prior & Posterior & Prior & Posterior \\
\hline \multirow{2}{*}{$Q$} & $\gamma$ & 1013.21 & 955.55 & $1002.70(81.77)$ & 144.65 & $100.55(30.49)$ \\
\hline & $\delta$ & 558.48 & 599.15 & $566.35(80.65)$ & 103.35 & $76.94(25.56)$ \\
\hline \multirow{2}{*}{$Z_{m}$} & $\mu_{Z m}$ & 55.03 & 54.93 & $55.00(70.00)$ & 0.25 & $0.190(24.00)$ \\
\hline & $\sigma_{\mathrm{Zm}}$ & 0.45 & 0.51 & $0.47(66.67)$ & 0.12 & $0.110(8.33)$ \\
\hline \multirow{2}{*}{$Z_{v}$} & $\mu_{Z v}$ & 50.19 & 50.11 & $50.17(75.00)$ & 0.21 & $0.165(21.43)$ \\
\hline & $\sigma_{Z v}$ & 0.38 & 0.45 & $0.39(85.71)$ & 0.16 & $0.121(24.38)$ \\
\hline \multirow{2}{*}{$\boldsymbol{K}_{s}$} & $\mu_{K s}$ & 27.80 & 25.24 & $26.95(66.80)$ & 6.45 & $5.40(16.28)$ \\
\hline & $\sigma_{K s}$ & 5.26 & 6.89 & $5.54(82.82)$ & 4.11 & $3.75(8.76)$ \\
\hline
\end{tabular}

It is evident that the method succeeds in moving the most likely values $c_{\theta}$ towards the corresponding MLE estimates $\hat{\theta}^{M L E}$ : actually, the values of $R_{\theta}^{\text {dist }}$ (3) range within $66.67 \%$ and $85.71 \%$. From the analysis of quantitative indicator $R_{\theta}$ (4) it can be seen that the method produces a consistent reduction in the area underlying the possibility distributions of the uncertain parameters: in particular, $R_{\theta}(4)$ ranges between $8.76 \%$ and $30.49 \%$. In addition, as expected, the strength of the approach in reducing epistemic uncertainty decreases with the size of the data set used to perform the Bayesian update. For example, the area $S_{\gamma}$ underlying the possibility distribution of $\gamma$ (Figure 2, top left) is reduced by $30.49 \%$ with the aid of a large data set of size $D_{1}=149$; on the contrary, the area $S_{\sigma_{K s}}$ underlying the possibility distribution of $\sigma_{K s}$ (Figure 2, bottom right) is reduced only by $8.76 \%$ by means of $D_{4}=5$ pieces of data.

In order to show the effect that the reduction of the epistemic uncertainty in the distribution parameters has on the maximal water level of the river $Z_{c}$ (i.e., the model output), Figure 3 shows the upper and lower CDFs $\bar{F}^{Z_{c}}\left(z_{c}\right)$ and $\underline{F}^{Z_{c}}\left(z_{c}\right)$ (i.e., the plausibility and belief functions, $P l^{Z_{c}}\left(\left(-\infty, z_{c}\right]\right)$ and $\mathrm{Bel}^{Z_{c}}\left(\left(-\infty, z_{c}\right]\right)$, respectively), of $Z_{c}$ obtained before (solid lines) and after the Bayesian update (dashed lines). Obviously, the gap between the plausibility and belief functions is larger before the Bayesian update: in particular, the 'prior' CDFs (solid lines) completely envelop the 'posterior' ones (dashed lines). This larger gap is explained by the larger area contained under the possibility distributions of the corresponding epistemically-uncertain parameters.

Then, in order to provide a fair and quantitative assessment of the approach adopted, proper indicators are computed. The final goal of the case study presented in Section 4 is to determine (i) the dike level necessary to guarantee a given flood return period or (ii) the flood risk for a given dike level. With respect to issue (i) above, the quantity of interest that is most relevant to the decision maker is the $\beta \cdot 100 \%$-th quantile of $Z_{c}$ (i.e., $Z_{c}^{\beta}$ ): this corresponds to the yearly maximal water level with a $\beta \cdot 100$-year return period. With respect to issue (ii) above, the quantity of interest is the probability that the maximal water level of the river $Z_{c}$ exceeds a given threshold $z^{*}$, i.e., $P\left[Z_{c} \geq z_{c}{ }^{*}\right]$ : in the present paper, $z_{c}^{*}=55.5 \mathrm{~m}$ (Table 2). The intervals $\left[\left[\bar{F}^{Z_{c}}\right]^{-1}(\beta)\right.$, $\left.\left[\underline{F}^{Z_{c}}\right]^{-1}(\beta)\right]$ for $Z_{c}^{\beta}, \beta=0.05,0.50$ and 0.95 , are [50.70, 51.67], [52.16, 53.46] and [54.13, 56.44], respectively, before the Bayesian update; instead, they are [50.90, 51.56], [52.38, 53.23] and [54.21, 55.87], respectively, after the update. Also, the intervals $\left[1-\bar{F}^{Z_{c}}\left(z_{c}^{*}\right), 1-\underline{F}^{Z_{c}}\left(z_{c}^{*}\right)\right]$ for $P\left[Z_{c}>z_{c}{ }^{*}\right]$ are $[0.0054$, 0.1092] and [0.0079, 0.0716], before and after the Bayesian update, respectively. Thus, the width of the intervals is reduced of $28.14-38.63 \%$.

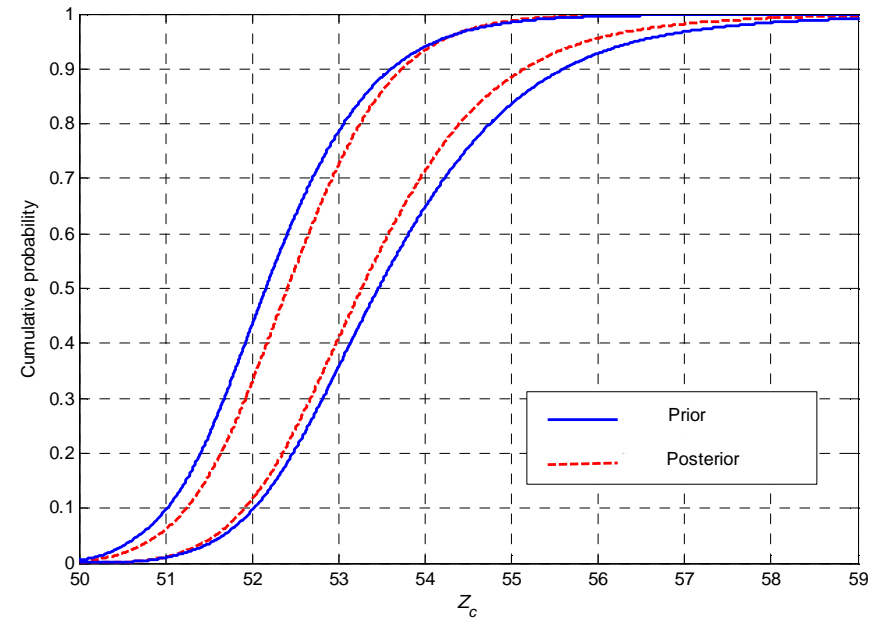

Figure 3. Plausibility and belief functions, $P l^{Z_{c}}\left(\left(-\infty, z_{c}\right]\right)$ and $B e l^{Z_{c}}\left(\left(-\infty, z_{c}\right]\right)$, of the maximal water level of the river $Z_{c}$ (i.e., the model output) before (solid lines) and after (dashed lines) the Bayesian update 
Table 2. Intervals for the $\beta \cdot 100$-th percentiles $Z_{c}^{\beta}, \beta=0.05$, 0.50 and 0.95 , of the maximal water level of the river $Z_{c}$ and for the exceedance probability $P\left[Z_{c}>z_{c}^{*}=55.5 \mathrm{~m}\right]$, before and after the Bayesian update. The percentage reduction in the width of the intervals is also shown in parentheses

\section{OUTPUT VARIABLE $\boldsymbol{Z}_{c}$}

\begin{tabular}{ccc}
\hline Indicator & Prior & $\begin{array}{c}\text { Posterior } \\
\text { (\% width reduction) }\end{array}$ \\
\hline $\boldsymbol{Z}_{c}^{\mathbf{0 . 0 5}}$ & {$[50.70,51.67]$} & {$[50.90,51.56](31.96)$} \\
\hline $\boldsymbol{Z}_{c}^{\mathbf{0 . 5 0}}$ & {$[52.16,53.46]$} & {$[52.38,53.23](34.62)$} \\
\hline $\boldsymbol{Z}_{c}^{\mathbf{0 . 9 5}}$ & {$[54.13,56.44]$} & {$[54.21,55.87](28.14)$} \\
\hline $\boldsymbol{P}\left[\boldsymbol{Z}_{c}>\boldsymbol{z}_{c}{ }^{*}\right]$ & {$[0.8908,0.9946]$} & {$[0.9284,0.9921](38.63)$} \\
\hline
\end{tabular}

A fundamental remark has to be done with respect to the results obtained. If on one side a consistent reduction in the epistemic uncertainty is in general desirable in decision making processes related to risk assessment problems (since it significantly increases the analyst's confidence in the decisions), on the other side this reduction must be coherent with the amount of information available. In this view, an objection may arise in the present case: is the remarkable strength of the approach in reducing epistemic uncertainty (even with very few pieces of data) fully justified by such a small amount of data? In other words, is this considerable reduction of epistemic uncertainty coherent with the strength of the experimental evidence or is it too optimistic? With respect to that, it has to be admitted that the uncertainty reduction power of the purely possibilistic approach is strongly dependent on the shape of an artificially constructed possibilistic likelihood that could in principle bias the analysis. However: (i) in the present paper, this possibilistic function is very closely related to the classical, purely probabilistic one (which is theoretically wellgrounded) by a simple and direct operation of normalization that preserves the "original structure" of the experimental evidence; (ii) in general, a probability-to-possibility transformation (properly performed according to the rules of possibility theory) always introduces additional artificial epistemic uncertainty into the analysis, i.e., it does not artificially reduce it (because it replaces a single probabilistic distribution by a family of distributions) (Flage et al. 2013). On the basis of considerations (i) and (ii) above, it seems unlikely that the purely possibilistic approach may produce results that are dangerously over-optimistic.

\section{CONCLUSIONS}

In this paper, we have considered a purely possibilistic counterpart of the classical probabilistic Bayes' theorem for the update of the possibilistic parameters of aleatory probability distributions, with exemplification on a case study concerning the riskbased design of a flood protection dike.

The findings of the work show that the strength of the approach in reducing epistemic uncertainty by means of data is considerable: this is important in decision making processes since reducing epistemic uncertainty significantly increases the analyst confidence in the decisions.

However, it has to be remarked that the construction of a possibilistic likelihood required by the method still represents an issue to be tackled from both the theoretical and practical viewpoint in order to avoid introducing biases in the analysis and to suggest the application of the approach for real risk assessment problems: with respect to that, future research will be devoted to the investigation of additional methods, either resorting to probabilitypossibility transformations or building possibilistic functions directly from rough experimental data.

\section{REFERENCES}

Aven, E. \& Zio, E. 2011. Some considerations on the treatment of uncertainties in risk assessment for practical decision making, Reliab Eng Syst Saf 96(1): 64-74.

Baudrit, C. \& Dubois, D. 2006. Practical Representations of Incomplete Probabilistic Knowledge. Computational Statistics \& Data Analysis 51(1): 86-108.

Baudrit, C., Dubois, D. \& Perrot, N. 2008. Representing parametric probabilistic models tainted with imprecision. Fuzzy Sets and System 159(15): 1913-1928.

Flage, R., Baraldi, P., Zio, E., Aven, T. 2013. Probability and possibility-based representations of uncertainty in fault tree analysis. Risk Analysis 33(1): 121-33.

Kelly, D.L. \& Smith, C.L, 2011. Bayesian Inference for Probabilistic Risk Assessment: A Practitioner's Guidebook. London, UK: Springer-Verlag.

Lapointe S. \& Bobeè B., 2000. Revision of possibility distributions: A Bayesian inference pattern. Fuzzy Sets and Systems 116: 119-140.

Le Duy, T.D., Vasseur, D., Couplet, M., Dieulle, L., Bérenguer, C., 2011. A study on updating belief functions for parameter uncertainty representation in Nuclear Probabilistic Risk Assessment. In F. Coolin, G. De Cooman, T. Fetz, M. Oberguggenberger (Eds.), Proceedings of the 7th International Symposium on Imprecise Probability: Theories and Applications, 25-28 July 2011, Innsbruck, Austria: 247256. Innsbruck, Austria: SIPTA.

Limbourg, P. \& de Rocquigny, E. 2010. Uncertainty analysis using evidence theory - confronting level-1 and level-2 approaches with data availability and computational constraints. Reliability Engineering and System Safety 95(5): 550-564.

Serrurier, M. \& Prade, H., 2011. Maximum-Likelihood Principle For Possibility Distributions Viewed As Families Of Probabilities. 2011 IEEE International Conference on Fuzzy Systems June 27-30, 2011, Taipei, Taiwan: 29872993.

Smets, P. 1993. Belief Functions: The Disjunctive Rule of Combination and the Generalized Bayesian Theorem. International Journal of Approximate Reasoning 9: 1-35.

Stein M., M. Beer, V. Kreinovich, 2013. Bayesian Approach for Inconsistent Information. Information Sciences: http://dx.doi.org/10.1016/j.ins.2013.02.024.

USNRC, 2009. Guidance on the Treatment of Uncertainties Associated with PRAs in Risk-Informed Decision Making. NUREG-1855, US Nuclear Regulatory Commission, Washington, DC. 